Overall, satisfaction with the TC among the prisoners was very high ( $80 \%$ good or excellent). Moreover, this is very cost effective with reduced cost of prisoner movement (Est $£ 500 /$ hospital visit). SVR and adherence data will be presented.

Conclusions A universal offer of BBV testing to prisoners at reception to prison can substantially increase testing rates and lead to many new diagnoses of HCV. Prison telemedicine clinics with nurse-led in-reach offer a cost effective and efficient method of treating $\mathrm{HCV}$ in the prison environment.

\section{PTU-117 THE IMPACT OF AN ACUTE JAUNDICE CLINIC AT A TERTIARY REFERRAL CENTRE}

James Morgan*, Denise Gocher, Esther Unitt. University Hospital Coventry And Warwickshire, Coventry, UK

\subsection{6/gutjnl-2018-BSGAbstracts.495}

Introduction Jaundice is not a particularly common presentation in general practice (56 in 100,000). However it often indicates a serious underlying condition (35\% malignancy) which requires urgent investigation via a 2 week wait referral. ${ }^{1}$ Various methods have been tried to expedite these referrals including a rapid access hotline. ${ }^{2}$ and clinics. The experience and impact of an acute jaundice clinic providing prompt clinical, biochemical and radiological assessment is evaluated at a tertiary referral centre.

Methods The acute jaundice clinic provides open access biweekly clinics, following primary care referral, for clinical assessment, same day access to radiological investigations and prompt referral for hepato-pancreato-biliary (HPB) MDT discussion and, if required, biliary decompression. The primary goal is to ensure patients found to have HPB cancers are assessed quickly; the secondary aim is to avoid unnecessary admissions. This review will analyse appropriateness of referral, timing of investigations, diagnoses made and subsequent patient outcomes. Data was collected contemporaneously and supplemented with online patient records. This included patient age, bilirubin level, referral date and date of clinic appointment, timeliness of radiological investigations, final diagnosis, date of discussion at HPB MDT and malignant patient outcomes.

Results Data analysis was completed for all patients seen in the jaundice clinic over a 3 year period (2015-2017). In total, 291 patients were referred with a median age of 68 years (range 18-96 years). 245 (84.2\%) of these were deemed appropriate to be seen with $172(70 \%)$ clinically jaundiced at the time of review. Median time from GP referral to jaundice clinic review was 5 days (range 1-33 days). 209 (85.3\%) of the patients were managed in the outpatient setting. The main diagnoses made following jaundice clinic are shown in figure 1 .

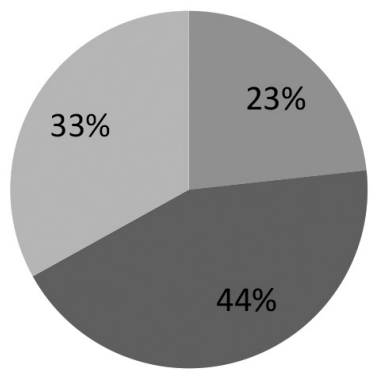

\section{Malignant \\ Gall stone related}

Other

Abstract PTU-117 Figure 1 Diagnoses made from jaundice clinic (percentage of patients)
For suspected malignant diagnoses, $90.4 \%$ had a CT on the day of clinic and were discussed at MDT, on average, 10 days (range 1-50 days) later. Outcome data was only available for 2016 and 2017 but in patients diagnosed with malignant biliary obstruction, 17/19 (89.5\%) had prompt biliary decompression with one of the remaining patients declining intervention. Only 4/19 (17.3\%) were eligible for potentially curative surgery and $5 / 19(26.3 \%)$ received palliative chemotherapy. One year survival was 50\% (9/18).

Conclusions The introduction of a jaundice clinic in a tertiary centre has been successful in providing timely review of jaundiced patients with high patient satisfaction. It has also allowed for prompt radiological assessment of potential malignant cases within 24 hours in more than $90 \%$ of cases with patients on average discussed in the HPB MDT within 10 days of jaundice clinic. The service has also proved extremely beneficial in avoiding admission in over $85 \%$ of patients. The remaining number admitted denotes the acute requirement for biliary decompression in a group of patients who often have significant co-morbidities. Disappointingly curative resection rates remain low in this cohort of patients, although this likely reflects the late stage of disease when jaundice is present and highlights the need for research into other predictors.

\section{REFERENCES}

1. Whitehead, MW, Hainsworth, I, Kingham, J. G. C. (2001). The causes of obvious jaundice in South West Wales: perceptions versus reality. Gut, 48(3),409-413.

2. Mitchell, J., Hussaini, H., McGovern, D., Farrow, R., Maskell, G., Dalton, H. (2002). Quality improvement report: The 'jaundice hotline' for the rapid assessment of patients with jaundice. BMJ: British Medical Journal, 325(7357),213.

\section{PTU-118 PRESENTING PROBLEMS AND OUTCOMES OF WOMEN WITH GYNAECOLOGICAL CANCER ATTENDING A SPECIALIST GI SYMPTOM CLINIC}

${ }^{1}$ Ann Muls*, ${ }^{1}$ Susan Lalondrelle, ${ }^{1}$ Alexandra Taylor, ${ }^{2}$ Christine Norton, ${ }^{3}$ Ailsa Hart, ${ }^{4}$ Jervoise Andreyev. ${ }^{1}$ The Royal Marsden NHS Foundation Trust, London, UK ${ }^{2}$ King's College London, London, UK ${ }^{3}$ Imperial College London, London, UK ${ }^{4}$ United Lincolnshire Hospitals NHS Trust, Lincoln, UK

\subsection{6/gutjnl-2018-BSGAbstracts.496}

Introduction Worldwide $1,470,900$ women are diagnosed yearly with a gynaecological cancer. Some women develop long-term changes in bowel function following treatment severely impacting on quality of life.

Methods A service evaluation collecting prospective data was approved by the organisation's $\mathrm{R}$ and $\mathrm{D}$ department. Intestinal symptoms were measured using a modified GSRS and impact on QoL assessed by VAS score $(0=$ worst QoL, $10=$ best QoL). The McNemar Chi-square and Wilcoxon signed rank tests were used to analyse changes in symptom burden between initial assessment to discharge from the service.

Results From April 2013 to March 2016, 235 women treated for gynaecological cancers attended the clinic, representing a fifth of those using the service. Fifteen declined further intervention and were excluded, leaving 220 for analysis. Women had been treated for cancer of the cervix (50\%), endometrium $(28 \%)$, ovary $(15 \%)$, vagina or vulva $(7 \%)$ cancer. Most women received multi-modal therapies: chemoradiation $(28 \%)$, surgery + radiotherapy (27\%), surgery +chemoradiation (22\%), surgery + chemotherapy (10\%). Median age was 57 years (range: 24-83). The median time between cancer diagnosis and referral to service was 4 years and 10 months 
(range: $6 \mathrm{~m}-47.5 \mathrm{y}$ ). Most troublesome symptoms at assessment were bowel frequency of 4 or more times a day $(88 \%)$, type 6 or 7 stool consistency (36\%), urgency to defaecate (31\%), faecal incontinence (21\%) and fatigue (25\%). Some women also reported urinary problems $(17 \%)$ and sexual concerns $(12 \%)$. Following a published algorithm, a median of 8 investigations were requested (range: 1-14): blood screen (97\%), gastroscopy (75\%), glucose hydrogen methane breath test (77\%), SeHCAT scan (71\%), faecal elastase (69\%), flexible sigmoidoscopy (52\%), colonoscopy (25\%) and abdominal Xray (18\%). A median of four factors contributing to symptoms were found (range: 1-9) and 68\% of women had more than three. The most common diagnoses were vitamin $\mathrm{D}$ deficiency (60\%), treated with replacement; SIBO (54\%), treated with antibiotics and bile acid malabsorption (52\%), treated with bile acid sequestrants and low fat diet. The median number of consultations was 4 (range: 1-17). Median quality of life improved from 4 at first assessment to 6 at discharge $(p<0.001)$; the reduction in urgency, incontinence, tenesmus, frequency, diarrhoea and fatigue was statistically significant $(\mathrm{p}<0.05)$.

Conclusions Bowel symptoms after cancer treatment affect many women. They can be discharged with symptom improvement using a systematic investigational and treatment approach. Earlier referral to specialist services is recommended.

\section{PTU-119 UPPER GASTROINTESTINAL HAEMORRHAGE: IS THERE A WEEKEND EFFECT? A REVIEW OF TWO DISTRICT GENERAL HOSPITALS}

${ }^{1}$ Duncan Napier* ${ }^{1}$ Rebecca Anderson, ${ }^{1}$ Giovanna Sheiybani, 2,35tephen Roberts, 2,3John Williams, 1,3 Jonathan Brown. 'Gloucestershire Hospitals NHS Foundation Trust, Gloucester, UK ${ }^{2}$ Swansea University Medical School, Swansea, UK ${ }^{3}$ Farr Institute of Health Informatics Research, Swansea University, Swansea, UK

\subsection{6/gutjnl-2018-BSGAbstracts.497}

Introduction Upper gastrointestinal haemorrhage (UGIH) is a common emergency presentation with a mortality reaching $10 \%{ }^{1}$ A 'weekend effect' has been described for UGIH with increased mortality rates for those admitted over a weekend. ${ }^{2}$ These studies typically utilise information from national databases to describe this effect whereas this study sought to examine if there was a reproducible 'weekend effect' at two district general hospitals.

Methods Retrospective data was extracted from the endoscopy database for both hospitals in 2014, identifying all patients with an indication suggestive of UGIH. The Trust coding database was used to identify all patients with an ICD-10 code suggestive of UGIH. These datasets were amalgamated and electronic admission records subsequently analysed to exclude inpatient UGIH. Admission and discharge documentation, endoscopy reports, GP records and bereavements records were reviewed to confirm day and time of admission and endoscopy, and survival to 30 days. Chi-squared test was used to compare mortality between groups.

Results There were 552 admissions for acute UGIH in 2014, 518 patients underwent an emergency endoscopy, 23 either did not have an endoscopy or had an outpatient endoscopy, and 11 notes were unavailable or incomplete and thus excluded. There was no statistically significant difference in 30 day mortality for those admitted on a weekday (Mon 0000 - Fri 2359) vs a weekend (11.05\% CI 7.98-14.79 vs
12.23\% CI 7.92-17.79, $\mathrm{p}=0.68 \mathrm{X}^{2}$ ). Neither was there a statistically significant difference in 30 day mortality for those admitted out of hours (1700-0859) compared to in hours (12.60\% CI $8.83-17.23$ vs $10.39 \%$ CI $7.07-14.59 \quad \mathrm{p}=0.43$ $\mathrm{X}^{2}$ ). Although not statistically significant, there was an increase in 30 day mortality for those requiring an out of hours procedure (1800-0759) compared to day time (23.08\% CI $14.89-33.09$ vs $8.64 \%$ CI $\quad 6.16-11.72 \quad \mathrm{p}=0.19$ $\mathrm{X}^{2}$ ).

Conclusions This study found no correlation between the day or time of admission for UGIH and 30 day mortality, suggesting that despite reduced levels of staffing and endoscopic activity over the weekend or out of hours this had no impact on 30 day mortality. This may be explained by appropriate patient selection for urgent endoscopy.

\section{PTU-120 IRON DEFICIENCY ANAEMIA IN RENAL CELL CARCINOMA}

Jeremy Nayagam*, Sun Ha, Lenka Barreto, Angelika Zang, Howard Curtis. Princess Royal University Hospital, King's College NHS Foundation Trust, London, UK

\subsection{6/gutjnl-2018-BSGAbstracts.498}

Introduction British Society of Gastroenterology (BSG) iron deficiency anaemia (IDA) investigation guidelines recommend urinalysis to screen for non-visible haematuria. If detected, renal ultrasound is recommended to exclude renal cell carcinoma (RCC). Data on IDA in RCC however suggests that haematuria is rarely detected. We sought to identify the prevalence of IDA in RCC, and the frequency of haematuria in this subgroup.

Methods All newly diagnosed RCC at a single NHS Trust over a 3 year period (1/1/13-31/12/15) were identified from a prospective database of the Urology multidisciplinary meeting (MDM). A retrospective analysis of electronic notes, MDM outcomes, clinic letters, laboratory results, radiology reports, endoscopy reports and histology was undertaken. Information on demographics, symptoms and investigation results were collected. IDA was defined by a combination of microcytosis, hypochromia and low ferritin at time of clinical review, or a diagnosis of IDA on enteral iron replacement prior to clinical review.

Results There were 163 cases of RCC in 162 patients, mean age 63.7 years, $35 \%$ female. 43 patients $(26.4 \%)$ were anaemic at diagnosis, IDA was present in 22 patients (13.5\%). $59 \%$ of IDA patients had previously undergone gastrointestinal (GI) endoscopy: one colonic melanoma; all others were normal or benign. Haematuria was reported in $22.1 \%$ of RCC patients, and $13.6 \%$ of those with IDA (3 patients). Of these 3 patients, 2 had visible haematuria and 1 had non-visible haematuria.

Conclusions Anaemia is commonly found in patients diagnosed with RCC at presentation. In our study group approximately half of anaemic patients were iron deficient. The majority of these had been previously investigated with GI endoscopy without a significant GI cause identified. Haematuria was not reported in most patients with IDA suggesting urinalysis may not be a suitable screening investigation. This adds further weight to the hypothesis that IDA associated with RCC is not from occult renal tract blood loss but from deposition in, or consumption by, the tumour itself. We propose that renal tract ultrasound is considered in all patients with IDA without significant GI pathology 